\title{
Lysophosphatidic Acid and Ion Channels as Molecular Mediators of Pain
}

\author{
Rebeca Juárez-Contreras, Tamara Rosenbaum* and Sara L. Morales-Lázaro * \\ División de Neurociencias, Instituto de Fisiología Celular, Departamento de Neurociencia Cognitiva, Universidad Nacional \\ Autónoma de México, Ciudad de México, Mexico
}

Lysophosphatidic acid or LPA is a phospholipid which has been extensively linked to the generation and maintenance of pain. Several ion channels have also been shown to participate in this pathological process but the link between LPA and these proteins in pain has just recently gained interest. In this respect, the field has advanced by determining the molecular mechanisms by which LPA promotes changes in the function of some ion channels. While some of the actions of LPA include modulation of signaling pathways associated to its specific receptors, other include a direct interaction with a region in the structure of ion channels to affect their gating properties. Here, we focus on the known effects of LPA on some transient receptor potential, sodium, potassium, and calcium channels. As the field moves forward, mechanisms are unveiled with the hope of understanding the underlying causes of pain in order to target these and control this pathophysiological state.

\section{OPEN ACCESS}

Edited by:

Javier Marquez,

Universidad de Málaga, Spain

Reviewed by:

Carolina Roza

University of Alcalá, Spain

Sangsu Bang,

Duke University, United States

Temugin Berta,

University of Cincinnati, United States

*Correspondence:

Tamara Rosenbaum trosenba@ifc.unam.mx Sara L. Morales-Lázaro saraluzm@ifc.unam.mx

Received: 27 September 2018 Accepted: 29 November 2018 Published: 12 December 2018

Citation:

Juárez-Contreras R, Rosenbaum T and Morales-Lázaro SL (2018) Lysophosphatidic Acid and lon Channels as Molecular Mediators of

Pain. Front. Mol. Neurosci. 11:462. doi: 10.3389/fnmol.2018.00462
Keywords: LPA, ion channel, pain, TRPV1, Nav1.8, K2P, Cav3.2

\section{INTRODUCTION}

Nociceptors are peripheral sensory neurons that respond to a wide diversity of harmful stimuli and transduce these stimuli into signals that reach the brain (Dubin and Patapoutian, 2010), leading to the subjective experience of pain defined as an "unpleased sensory and emotional experience associated with actual or potential tissue damage" (International Association of Pain Study).

Neuronal activity is initiated at these specialized neurons, with somas located at the dorsal root ganglia (DRG) or trigeminal ganglia (TG) (Dubin and Patapoutian, 2010). The axons of these neurons split into two branches with one innervating peripheral organs and the other projecting to the dorsal horn or to the brainstem (Figure 1). These nociceptors are classified into A $\delta$ fibers which are medium-size and lightly myelinated fibers with a conduction velocity of $\sim 5-30 \mathrm{~m} / \mathrm{s}$ or as small diameter unmyelinated $\mathrm{C}$ fibers that have lower conduction velocity $(\sim 0.4-1.4 \mathrm{~m} / \mathrm{s})$ (Dubin and Patapoutian, 2010).

Nociceptors contain a huge diversity of specialized membrane proteins such as G-proteincoupled receptors (GPCRs) that regulate neuronal physiology and ion channels that form highly regulated gates that control the influx of ions through the plasma membrane, resulting in changes of the membrane potential.

Noxious inputs trigger the activation of ion channels such as: acid sensing ion channels (ASIC), P2X purinoreceptors and members of the transient receptor potential (TRP) family, such as the TRPV1 (vanilloid) channel (Basbaum et al., 2009). Their activation leads to membrane depolarization initiating the first step of the pain pathway where noxious signals are converted into electrical messages (transduction) (McEntire et al., 2016). 


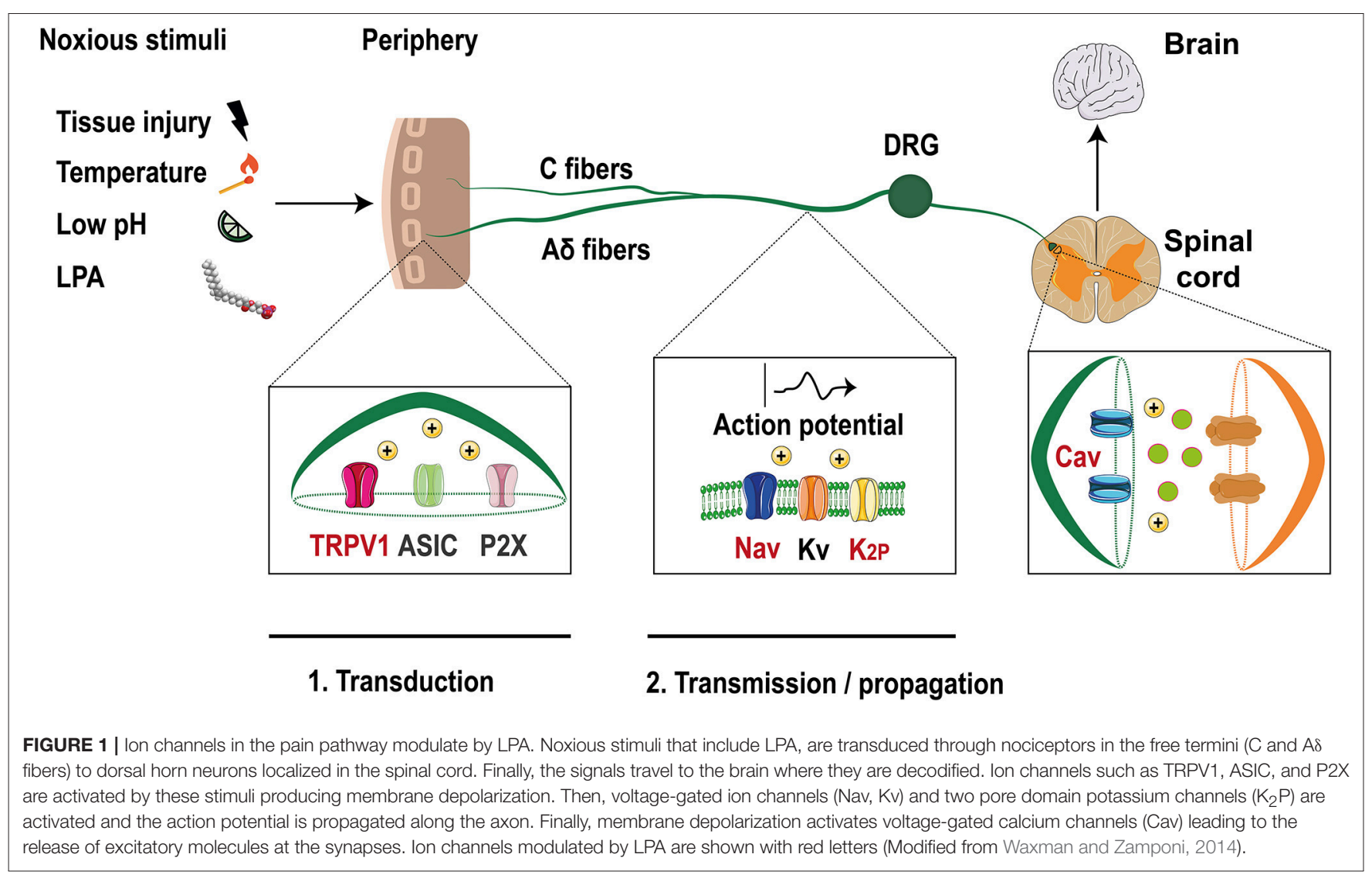

If the magnitude of depolarization is sufficient, voltage-gated sodium channels (Nav) are activated and an action potential (AP) is initiated and propagated along the axon (transmission) until it reaches the synaptic zone, where the activation of voltagegated calcium channels (Cav) produces the release of excitatory neurotransmitters (Sekiguchi et al., 2018). Furthermore, voltagegated potassium channels $(\mathrm{Kv})$ are expressed in nociceptors and their activation leads to membrane repolarization and hyperpolarization (i.e., two pore domain potassium channels, $\mathrm{K}_{2 \mathrm{P}}$ ), inhibiting the signaling pathway, and allowing the neurons to respond again (Ocana et al., 2004).

An endogenous chemical mediator of pain produced during tissue injury or inflammation states is lysophosphatidic acid (LPA) (Eichholtz et al., 1993), a phospholipid that regulates the expression/function of ion channels in the pain pathway.

LPA is composed of a glycerol backbone with an ester link to a phosphate head group and an acyl fatty tail (Figure 2). This tail is usually constituted by 16 to 20 carbons linked by single (saturated) or double (unsaturated) bonds giving rise to different LPA species (Gerrard and Robinson, 1989). LPA is mainly produced through autotaxin, an ectoenzyme that uses lysophospholipids as precursor molecules (Tokumura et al., 2002) or by enzymatic actions of phospholipases A1 or A2 (PLA1/2) using phosphatidic acid as substrate (Aoki et al., 2002).

LPA's biological effects are broad and include participation in cell growth, differentiation, proliferation, survival, motility, cytoskeleton changes, and pain (Yung et al., 2014). Classical
LPA actions are mediated by six specific GPCRs: LPA $1-6$ (Yung et al., 2014). These trigger different signaling pathways through $\mathrm{G} \alpha$ proteins $\left(\mathrm{G}_{\mathrm{i}}, \mathrm{G}_{\mathrm{q}}, \mathrm{G}_{12}\right.$, and $\left.\mathrm{G}_{\mathrm{s}}\right)$ producing different tissue responses such as in the nervous system, where LPA signaling has an important role in neuronal development and myelination (Yung et al., 2015). Importantly, activation of these receptors also leads to the generation of pain in fully-developed model animals (Inoue et al., 2004).

This review focuses on the effects of LPA on some ion channels expressed in sensory neurons and discusses the relevance of the regulation of these proteins by LPA in pain. In particular, we discuss the effects of LPA on TRPV1, Nav1.8, TRESK, TREK1, Cav3.2 channels, and the $\alpha 2 \delta 1$ subunit of Cav2.2 channels, which are the only channels shown to be regulated by LPA to date.

\section{ACTIVATION OF TRPV1 CHANNELS BY LPA}

Several noxious signals are converted into electrical ones through TRP ion channels. The better studied member of this family is TRPV1, which is widely expressed in small diameter neurons of the DRG and TG (Caterina et al., 1997) and is activated by temperatures $\geq 43^{\circ} \mathrm{C}$, extracellular acid and intracellular basic pHs, pungent chemical compounds, as capsaicin, resineferatoxin, allicin, and by some toxins (Szolcsanyi and Sandor, 2012). Moreover, some lipidic compounds produced during inflammation or tissue injury (i.e., anandamide, 


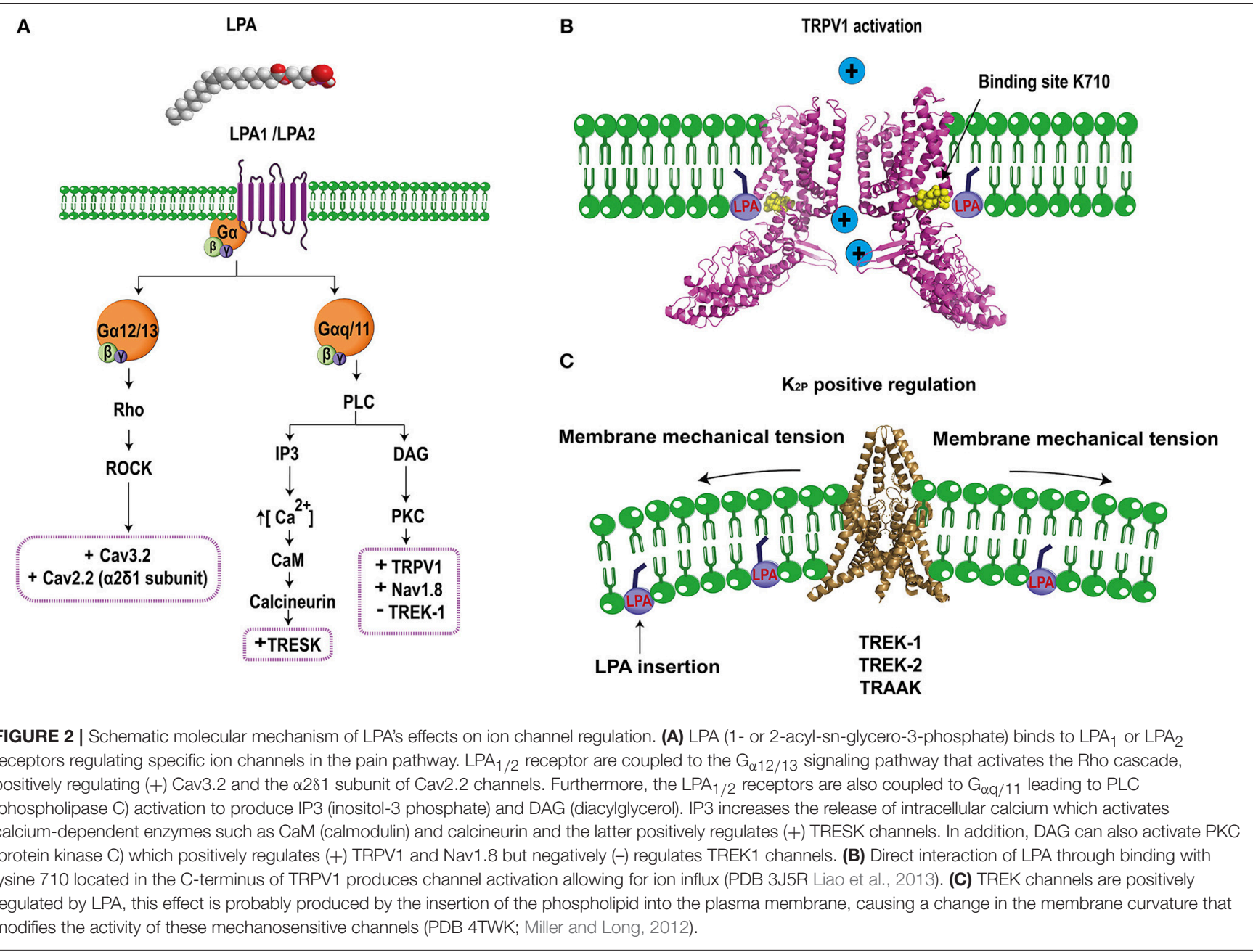

metabolites of lipoxygenase, and LPA) function as endogenous activators of TRPV1 producing pain (Morales-Lazaro et al., 2013).

The first connection between LPA and TRPV1 was described in rat DRG neurons, where the co-expression of this channel with the $\mathrm{LPA}_{1}$ receptor (associated to pain), was determined. Electrophysiological experiments in DRGs showed a dosedependent potentiation of capsaicin evoked currents by LPA, which were blocked by inhibitors of the $\mathrm{LPA}_{1}$ receptor and $\mathrm{PKC} \varepsilon$ (Figure 2A), demonstrating that downstream activation of PKC $\varepsilon$ by the $\mathrm{LPA}_{1}$ receptor pathway is crucial for TRPV1 sensitization (Pan et al., 2010).

Shortly after this, our group demonstrated the direct activation of TRPV1 by LPA and showed that this phospholipid produces acute pain through this channel when it is injected into the paws of mice (Nieto-Posadas et al., 2011). This painlike behavior produced by LPA was decreased in the TRPV1 knockout (KO) mice, strongly indicating that LPA requires TRPV1 to transduce this noxious input. In addition, by using a mouse model where the gene that encodes for the enzyme that dephosphorylates LPA (LPP3) was conditionally deleted and where LPA levels are augmented in the nervous system, we showed that these animals exhibited thermal hyperalgesia and increased pain, as compared to the wild-type (WT) mice (Nieto-Posadas et al., 2011).

Furthermore, we also demonstrated that LPA elicited action potential firing in WT mouse DRGs, whereas this electrical activity was not observed in DRG neurons from TRPV1 KO mice. We detailed TRPV1 activation by LPA in excised membranes patches from TRPV1-expressing HEK293 cells and found that TRPV1 currents were generated upon intracellular and extracellular LPA application (Nieto-Posadas et al., 2011) and that this phospholipid produced a different conformational change to that produced by capsaicin, allowing for a larger conductance state (Canul-Sanchez et al., 2018).

We next assessed the role of LPA receptors on the activation of TRPV1 and showed that these where not responsible for activation of TRPV1. These data led us to propose and to demonstrate a direct interaction between LPA and TRPV1 (Figure 2B). By combining mutagenesis, in vitro interaction assays and electrophysiology, we showed that LPA binds to a lysine located on the carboxy- end of TRPV1 (K710), (Nieto-Posadas et al., 2011), a binding site shared with PIP2 (an anionic lipid that regulates the function of 
TRPV1) (Ufret-Vincenty et al., 2011). Altogether, these results demonstrated that LPA produced acute pain through a direct activation of TRPV1 channel.

The structural determinants of LPA required for activation of TRPV1 are: a monounsaturated long acyl chain (18 carbons for natural phospholipids and from 18 to 20 carbons for the synthetic analogs) and an anionic head-group (Morales-Lazaro et al., 2014).

Another TRP channel that is activated by LPA is TRPA1 in which the phospholipid directly interacts with positively-charged residues located in the amino-end (K672-K673) and in the carboxy-end (K977-R978) of the channel (Kittaka et al., 2017). However, that study reported that the physiological response to LPA injection in mice is mostly an itch behavior and not one of pain.

\section{LPA AS A REGULATOR OF SODIUM CURRENTS ON SENSORY NEURONS}

As mentioned before, the action potential is initiated and propagated along the axons of primary neurons due to the electrical activity of Nav channels. These are formed by $\alpha$ and $\beta$ subunits with nine independent genes coding for the $\alpha$ subunits that produce the Nav1.1-1.9 channels, among which Nav1.7-1.9 have been associated to pain (Theile and Cummins, 2011).

A relationship between LPA-dependent pain and these channels was determined in rat small DRG neurons, where sodium currents were shown to exhibit increased tetrodotoxin (TTX)-resistant currents in the presence of LPA (Seung Lee et al., 2005). These neurons express TTX-resistant Nav1.8 and Nav1.9 channels (Ho and O'Leary, 2011); thus, the data suggested that LPA positively regulates these currents essential for the pain pathway.

Experiments also showed that intrathecal injection of LPA upregulates the expression of the Nav1.8 channel together with the $L^{2} A_{1}$ receptor in rat DRG neurons, effects that were blocked when LPA was co-injected with an antagonist of the $\mathrm{LPA}_{1}$ receptor (Pan et al., 2016). Additional experiments performed on small DRG neurons, where the membrane potential was maintained at $-60 \mathrm{~V}$ (to inhibit currents produced by Nav1.9 channels) and LPA was perfused, showed Nav1.8current potentiation indicating that the activity of this specific channel is positively regulated by LPA (Pan et al., 2016).

LPA effects on sodium currents were also reported in a rat bone cancer model, where the animals exhibit severe hyperalgesia, displaying $\mathrm{LPA}_{1}$ receptor upregulation and showing a higher percentage of LPA-sensitive C-fibers than in control animals (Pan et al., 2016). In this model, the expression of Nav1.8 channels is upregulated and these are widely co-localized with $\mathrm{LPA}_{1}$ in DRG neurons. Moreover, animals treated with an inhibitor of the $\mathrm{LPA}_{1}$ receptor, exhibited a decrease in the expression of Nav1.8 channels and partial attenuation of mechanical hyperalgesia (Pan et al., 2016).

Evidences obtained with this model and through intrathecal LPA injection demonstrated a crosstalk between Nav1.8 channels and the $\mathrm{LPA}_{1}$ receptor, suggesting the participation of a downstream pathway (Figure 2A). Further experiments using an inhibitor of PKCE showed that Nav1.8 current potentiation are mediated through this intracellular signaling pathway (Pan et al., 2016).

\section{TWO PORE DOMAIN POTASSIUM CHANNELS $\left(K_{2 P}\right)$ ARE TARGETS OF LPA}

The resting membrane potential of cells is controlled by potassium channels, since these facilitate repolarization and hyperpolarization of the membrane and regulate pain (Du and Gamper, 2013). Some members of the potassium channel family are expressed in nociceptors: $\mathrm{K}_{\mathrm{V}}$, calcium activated potassium $\left(\mathrm{K}_{\mathrm{Ca}}\right)$ and two pore domain potassium $\left(\mathrm{K}_{2 \mathrm{P}}\right)$ channels (Du and Gamper, 2013). Although, some reports have shown that LPA regulates their function, trafficking or expression, few studies have shown their relationship to the pain pathway.

For example, it has been reported that some $\mathrm{K}_{2 \mathrm{P}}$ channels are targets of LPA, contributing to the modulation of pain. TRESK, TREK1-2, TRAAK, TALK1, TASK1-2 channels are expressed in DRG and the TRESK channel is the most abundant $K_{2 P}$ in DRG neurons (Dobler et al., 2007). The latter importantly contributes to the background $\mathrm{K}^{+}$conductance in nociceptors and plays a role in the excitability of these neurons. TRESK's role in nociception has been demonstrated in DRG neurons from mice lacking its expression where less current input is needed to elicit action potentials (Cohen et al., 2009) and in TG neurons that over-express this channel and display reduced excitability as well as a significant reduction in the spike frequency in response to capsaicin (Guo and Cao, 2014). The TRESK channel is co-expressed with the $\mathrm{LPA}_{2}$ receptor and TRPV1 ion channels in small diameter mice DRG neurons (Kollert et al., 2015). A direct relationship between TRESK channels and the standing outward current evoked by LPA has been shown in Xenopus oocytes that co-express TRESK channels and the $\mathrm{LPA}_{2}$ receptor, where an increase in basal potassium currents was observed upon LPA application (Kollert et al., 2015). The experiments showed that LPA effects were significantly reduced if the cells were preincubated with an inhibitor of phospholipase $\mathrm{C}$ and eliminated by using a TRESK channel where the calcineurin binding site was mutated. These results indicated that a GPCR signaling pathway facilitates LPA's effects on TRESK (Figure 2A). Additionally, experiments in DRG neurons showed that LPA elicits background standing outward currents through TRESK-channel activation since DRG neurons from TRESK KO mice lost these currents, whereas the inward currents produced through TRPV1's activation by LPA were maintained (Kollert et al., 2015). Furthermore, DRG neurons from TRESK KO mice displayed increases in spike frequency upon LPA application, exhibiting enhanced excitability (Kollert et al., 2015). In this case, LPA has a complex effect by positively modulating TRESK channels and functioning as a pain inhibitor while activating TRPV1 that is also expressed in the same neurons producing pain. It could be hypothesized that activation of TRESK channels serves as a compensation mechanism that 
decreases depolarization induced through the activation of other channels (i.e., TRPV1). It is yet to be clarified under which scenario LPA can effectively produce pain by bypassing the inhibitory activity of TRESK channels. In this respect, it is known that the $\mathrm{LPA}_{2}$ receptor desensitizes and, since the activation of TRESK channels depends on the signaling pathway of this GPCR, it is possible that the pain inhibiting role of TRESK channels is overcome by the activation of TRPV1 by LPA finally resulting in pain.

Another $\mathrm{K}_{2 \mathrm{P}}$ channel with moderate expression in DRG neurons is the TREK-1 channel, which is regulated by mechanic, chemical, and thermal stimuli (Maingret et al., 1999). Similar to TRESK, it has been reported that LPA positively regulates TREK-1. The first report was obtained from COS cells transiently expressing TREK-1 where inside-out excised patches exposed to LPA exhibited TREK-1's activation, contrary to COS cells without TREK-1 expression where LPA intracellular application failed to produce currents (Chemin et al., 2005).

TREK-1 is characterized by its intrinsic voltage-dependence and its low activation at atmospheric pressure, properties that are reverted by the intracellular presence of LPA. TREK-2 and TRAAK channels are also regulated at atmospheric pressure in the presence of LPA (Chemin et al., 2005). Although, the molecular mechanism of activation of TREK-1, TREK-2, and TRAAK by LPA is still unresolved, "it has been suggested that LPA affects membrane-curvature (Figure 2C), resulting in modulation of the function of these mechanosensitive channels" (Chemin et al., 2005).

In contrast to the effect described above where intracellular LPA activates TREK-1 and which possibly depends on changes in the membrane curvature, inhibitory LPA effects on TREK-1's activation have also been reported in Xenopus oocytes expressing human TREK-1 channels when LPA is now extracellularly applied (Cohen et al., 2009). In this case, LPA extracellularly binds to a GPCR and triggers activation of the PLC producing the inhibition of TREK-1 (Figure 2A). When serines S315 and S348 in TREK-1 are substituted by alanines, the channels are not phosphorylated by PKC and renders them resistant to LPA's effects (Cohen et al., 2009). Although the dependence of the pain response upon inhibition of TREK-1 by LPA has not been demonstrated yet, it can be hypothesized if the channel is inhibited by LPA, a possible physiological consequence on the pain pathway would be a decrease in the threshold to pain and, under this scenario, LPA would function as an algogenic molecule through modulation of TREK-1 activity under pathological situations where LPA's extracellular levels are increased.

\section{VOLTAGE-GATED CALCIUM CHANNELS ARE TARGETS OF LPA'S SIGNALING PATHWAYS}

When the action potential in the pain pathway arrives to synaptic nerve terminals of primary neurons, depolarization-induced opening of Cav channels occurs (Sekiguchi et al., 2018). CaV channels are classified as high- or low-voltage-activated (HVA and LVA, respectively). They share a structural topology similar to that of Nav channels. Additionally, HVA calcium channels (Cav1.2-1.4 and Cav2.1-2.3) are composed by accessory subunits $(\alpha 2 \delta$ and $\beta$ ), which regulate their cell surface density (Dolphin, 2016).

Several reports have shown that intrathecal LPA injection in mice produces the same effects as observed in the mice with partial sciatic nerve injury, a neuropathic pain model (Ueda, 2006). These mice display mechanical allodynia, thermal hyperalgesia, and demyelination (Inoue et al., 2004). Moreover, LPA injection and nerve injury triggers up-regulation of the $\alpha 2 \delta 1$ subunit of HVA calcium channels in DRG neurons. This up-regulation can be avoided in the $\mathrm{LPA}_{1}$ receptor null-mice or by using a RhoA inhibitor (Inoue et al., 2004). Thus, these data demonstrate that LPA positively regulates $\alpha 2 \delta 1$ expression, a subunit important for Cav2.2, which is abundantly expressed in nociceptors (N-type calcium channels; Figure 2A). Such effects are mediated through the $\mathrm{LPA}_{1}$ receptor, which probably enhances the excitability of sensory neurons causing neuropathic pain. Mice lacking $\mathrm{N}$-type calcium-channel expression show resistance to developing neuropathic pain symptoms (Saegusa et al., 2001), in contrast to the effects of LPA that positively regulates the auxiliary subunit $\alpha 2 \delta 1$ of this channel resulting in pain (Inoue et al., 2004).

Cav3.2, a LVA calcium channel is expressed in small DRG neurons (Rose et al., 2013) and it has been shown that mice intrathecally-treated with antisense oligonucleotides against Cav3.2 display antinociceptive, anti-hyperalgesic, and antiallodynic effects (Bourinet et al., 2005). This channel is also regulated by LPA and it was demonstrated in rat DRG neurons that T-type currents following extracellular LPA application are increased through a mechanism dependent on the GPCR signaling pathway (Figure 2A) since the effect is blocked when the neurons are pre-treated with a ROCK inhibitor (Iftinca et al., 2007).

Positive regulation of Cav channels in nociceptors guarantees the release of excitatory amino acids (glutamate and aspartate) and/or neuropeptides (substance P) at their synapses with dorsal horn neurons that project to the brain (Zamponi et al., 2009); thus, Cavs play an important role in the transmission of harmful signals that may be regulated by LPA.

\section{CONCLUSION}

Regulation of ion channels by endogenous molecules is key to the generation and maintenance of pain. The elucidation of the nature of these molecules and the molecular mechanisms by which they produce pain is fundamental to achieve tools to understand and control pathological processes associated to their functions. Much work is still needed to address questions on which other ion channels are regulated by this phospholipid and on how the native systems where these are expressed are effectively regulated by LPA and other substances whose levels are increased under pathological situations. For example, there is scarce information as to how this phospholipid regulates $\mathrm{P} 2 \mathrm{X} 3$ receptors that have been shown to participate in pain 
(Burnstock, 2016). Only one study shows that LPA can regulate the activity of $\mathrm{P} 2 \mathrm{X} 3$ receptors through an LPA1-mediated signaling pathway in bonce cancer pain in rats (Wu et al., 2016).

It is also important to consider that some pathophysiological conditions may produce sensitization of nociceptors and leading to a shift in the pain threshold in response to LPA (Gold and Gebhart, 2010).

\section{AUTHOR CONTRIBUTIONS}

RJ-C, TR, and SLM-L conceived and wrote this manuscript.

\section{REFERENCES}

Aoki, J., Taira, A., Takanezawa, Y., Kishi, Y., Hama, K., Kishimoto, T., et al. (2002). Serum lysophosphatidic acid is produced through diverse phospholipase pathways. J. Biol. Chem. 277, 48737-48744. doi: 10.1074/jbc.M206812200

Basbaum, A. I., Bautista, D., M., Scherrer, G., and Julius, D. (2009). Cellular and molecular mechanisms of pain. Cell 139, 267-284. doi: 10.1016/j.cell.2009.09.028

Bourinet, E., Alloui, A., Monteil, A., Barrere, C., Couette, B., Poirot, O., et al. (2005). Silencing of the Cav3.2 T-type calcium channel gene in sensory neurons demonstrates its major role in nociception. EMBO J. 24, 315-324. doi: 10.1038/sj.emboj.7600515

Burnstock, G. (2016). Purinergic mechanisms and pain. Adv. Pharmacol. 75, 91-137. doi: 10.1016/bs.apha.2015.09.001

Canul-Sanchez, J. A., Hernandez-Araiza, I., Hernandez-Garcia, E., Llorente, I., Morales-Lazaro, S. L., Islas, L. D., et al. (2018). Different agonists induce distinct single-channel conductance states in TRPV1 channels. J. Gen. Physiol. 150, 1735-1746. doi: 10.1085/jgp.201812141

Caterina, M. J., Schumacher, M. A., Tominaga, M., Rosen, T. A., Levine, J. D., and Julius, D. (1997). The capsaicin receptor: a heat-activated ion channel in the pain pathway. Nature 389, 816-824. doi: 10.1038/39807

Chemin, J., Patel, A., Duprat, F., Zanzouri, M., Lazdunski, M., and Honore, E. (2005). Lysophosphatidic acid-operated K+ channels. J. Biol. Chem. 280, 4415-4421. doi: 10.1074/jbc.M408246200

Cohen, A., Sagron, R., Somech, E., Segal-Hayoun, Y., and Zilberberg, N. (2009). Pain-associated signals, acidosis and lysophosphatidic acid, modulate the neuronal K(2P)2.1 channel. Mol. Cell. Neurosci. 40, 382-389. doi: 10.1016/j.mcn.2008.12.004

Dobler, T., Springauf, A., Tovornik, S., Weber, M., Schmitt, A., Sedlmeier, R., et al. (2007). TRESK two-pore-domain $\mathrm{K}+$ channels constitute a significant component of background potassium currents in murine dorsal root ganglion neurones. J. Physiol. 585 (Pt 3), 867-879. doi: 10.1113/jphysiol.2007.1 45649

Dolphin, A. C. (2016). Voltage-gated calcium channels and their auxiliary subunits: physiology and pathophysiology and pharmacology. J. Physiol. 594, 5369-5390. doi: 10.1113/JP272262

$\mathrm{Du}, \mathrm{X}$., and Gamper, N. (2013). Potassium channels in peripheral pain pathways: expression, function and therapeutic potential. Curr. Neuropharmacol. 11, 621-640. doi: 10.2174/1570159X113119990042

Dubin, A. E., and Patapoutian, A. (2010). Nociceptors: the sensors of the pain pathway. J. Clin. Invest. 120, 3760-3772. doi: 10.1172/JCI42843

Eichholtz, T., Jalink, K., Fahrenfort, I., and Moolenaar, W. H. (1993). The bioactive phospholipid lysophosphatidic acid is released from activated platelets. Biochem. J. 291 (Pt 3), 677-680.

Gerrard, J. M., and Robinson, P. (1989). Identification of the molecular species of lysophosphatidic acid produced when platelets are stimulated by thrombin. Biochim. Biophys. Acta 1001, 282-285.

Gold, M. S., and Gebhart, G. F. (2010). Nociceptor sensitization in pain pathogenesis. Nat. Med. 16, 1248-1257. doi: 10.1038/nm.2235

\section{FUNDING}

This work was supported by grants from Dirección General de Asuntos del Personal Académico (DGAPA)Programa de Apoyo a Proyectos de Investigación e Innovación Tecnológica (PAPIIT) Grand IA202717 and by Estímulos a Investigaciones Médicas Miguel Alemán Valdés to SLM-L and Consejo Nacional de Ciencia y Tecnología (CONACyT) Grand A1-S-8760. CONACyT Fronteras en la Ciencia No. 77, and DGAPA-PAPIIT grand IN200717 to TR. RJ-C received scholarship 488802 from CONACyT.

Guo, Z., and Cao, Y. Q. (2014). Over-expression of TRESK K(+) channels reduces the excitability of trigeminal ganglion nociceptors. PLoS ONE 9:e87029. doi: 10.1371/journal.pone.0087029

Ho, C., and O'Leary, M. E. (2011). Single-cell analysis of sodium channel expression in dorsal root ganglion neurons. Mol. Cell. Neurosci. 46, 159-166. doi: 10.1016/j.mcn.2010.08.017

Iftinca, M., Hamid, J., Chen, L., Varela, D., Tadayonnejad, R., Altier, C., et al. (2007). Regulation of T-type calcium channels by Rho-associated kinase. Nat. Neurosci. 10, 854-860. doi: 10.1038/nn1921

Inoue, M., Rashid, M. H., Fujita, R., Contos, J. J., Chun, J., and Ueda, H. (2004). Initiation of neuropathic pain requires lysophosphatidic acid receptor signaling. Nat. Med. 10, 712-718. doi: 10.1038/nm1060

Kittaka, H., Uchida, K., Fukuta, N., and Tominaga, M. (2017). Lysophosphatidic acid-induced itch is mediated by signalling of LPA5 receptor, phospholipase D and TRPA1/TRPV1. J. Physiol. 595, 2681-2698. doi: 10.1113/JP273961

Kollert, S., Dombert, B., Doring, F., and Wischmeyer, E. (2015). Activation of TRESK channels by the inflammatory mediator lysophosphatidic acid balances nociceptive signalling. Sci. Rep. 5:12548. doi: 10.1038/srep 12548

Liao, M., Cao, E., Julius, D., and Cheng, Y. (2013). Structure of the TRPV1 ion channel determined by electron cryo-microscopy. Nature 504, 107-112. doi: $10.1038 /$ nature 12822

Maingret, F., Patel, A. J., Lesage, F., Lazdunski, M., and Honore, E. (1999). Mechano- or acid stimulation, two interactive modes of activation of the TREK-1 potassium channel. J. Biol. Chem. 274, 26691-26696.

McEntire, D. M., Kirkpatrick, D. R., Dueck, N. P., Kerfeld, M. J., Smith, T. A., Nelson, T. J., et al. (2016). Pain transduction: a pharmacologic perspective. Expert Rev. Clin. Pharmacol. 9, 1069-1080. doi: 10.1080/17512433.2016.1183481

Miller, A. N., and Long, S. B. (2012). Crystal structure of the human two-pore domain potassium channel K2P1. Science 335, 432-436. doi: 10.1126/science.1213274

Morales-Lazaro, S. L., Serrano-Flores, B., Llorente, I., Hernandez-Garcia, E., Gonzalez-Ramirez, R., Banerjee, S., et al. (2014). Structural determinants of the transient receptor potential 1 (TRPV1) channel activation by phospholipid analogs. J. Biol. Chem. 289, 24079-24090. doi: 10.1074/jbc.M114.572503

Morales-Lazaro, S. L., Simon, S. A., and Rosenbaum, T. (2013). The role of endogenous molecules in modulating pain through transient receptor potential vanilloid 1 (TRPV1). J. Physiol. 591, 3109-3121. doi: 10.1113/jphysiol.2013.251751

Nieto-Posadas, A., Picazo-Juarez, G., Llorente, I., Jara-Oseguera, A., MoralesLazaro, S., Escalante-Alcalde, D., et al. (2011). Lysophosphatidic acid directly activates TRPV1 through a C-terminal binding site. Nat. Chem. Biol. 8, 78-85. doi: $10.1038 /$ nchembio.712

Ocana, M., Cendan, C. M., Cobos, E. J., Entrena, J. M., and Baeyens, J. M. (2004). Potassium channels and pain: present realities and future opportunities. Eur. J. Pharmacol. 500, 203-219. doi: 10.1016/j.ejphar.2004.07.026

Pan, H. L., Liu, B. L., Lin, W., and Zhang, Y. Q. (2016). Modulation of Nav1.8 by Lysophosphatidic Acid in the Induction of Bone Cancer Pain. Neurosci. Bull. 32, 445-454. doi: 10.1007/s12264-016-0060-7 
Pan, H. L., Zhang, Y. Q., and Zhao, Z. Q. (2010). Involvement of lysophosphatidic acid in bone cancer pain by potentiation of TRPV1 via PKCepsilon pathway in dorsal root ganglion neurons. Mol. Pain 6:85. doi: 10.1186/1744-8069-6-85

Rose, K. E., Lunardi, N., Boscolo, A., Dong, X., Erisir, A., Jevtovic-Todorovic, V., and Todorovic, S. M. (2013). Immunohistological demonstration of CaV3.2 Ttype voltage-gated calcium channel expression in soma of dorsal root ganglion neurons and peripheral axons of rat and mouse. Neuroscience 250, 263-274. doi: 10.1016/j.neuroscience.2013.07.005

Saegusa, H., Kurihara, T., Zong, S., Kazuno, A., Matsuda, Y., Nonaka, T., et al. (2001). Suppression of inflammatory and neuropathic pain symptoms in mice lacking the N-type Ca2+ channel. EMBO J. 20, 2349-2356. doi: $10.1093 /$ emboj/20.10.2349

Sekiguchi, F., Tsubota, M., and Kawabata, A. (2018). Involvement of voltage-gated calcium channels in inflammation and inflammatory pain. Biol. Pharm. Bull. 41, 1127-1134. doi: 10.1248/bpb.b18-00054

Seung Lee, W., Hong, M. P., Hoon Kim, T., Kyoo Shin, Y., Soo Lee, C., Park, M., et al. (2005). Effects of lysophosphatidic acid on sodium currents in rat dorsal root ganglion neurons. Brain Res. 1035, 100-104. doi: 10.1016/j.brainres.2004.12.026

Szolcsanyi, J., and Sandor, Z. (2012). Multisteric TRPV1 nocisensor: a target for analgesics. Trends Pharmacol. Sci. 33, 646-655. doi: 10.1016/j.tips.2012.09.002

Theile, J. W., and Cummins, T. R. (2011). Recent developments regarding voltage-gated sodium channel blockers for the treatment of inherited and acquired neuropathic pain syndromes. Front. Pharmacol. 2:54. doi: 10.3389/fphar.2011.00054

Tokumura, A., Majima, E., Kariya, Y., Tominaga, K., Kogure, K., Yasuda, K., et al. (2002). Identification of human plasma lysophospholipase $\mathrm{D}$, a lysophosphatidic acid-producing enzyme, as autotaxin, a multifunctional phosphodiesterase. J. Biol. Chem. 277, 39436-39442. doi: $10.1074 /$ jbc.M205623200

Ueda, H. (2006). Molecular mechanisms of neuropathic pain-phenotypic switch and initiation mechanisms. Pharmacol. Ther. 109, 57-77. doi: 10.1016/j.pharmthera.2005.06.003
Ufret-Vincenty, C. A., Klein, R. M., Hua, L., Angueyra, J., and Gordon, S. E. (2011). Localization of the PIP2 sensor of TRPV1 ion channels. J. Biol. Chem. 286, 9688-9698. doi: 10.1074/jbc.M110. 192526

Waxman, S. G., and Zamponi, G. W. (2014). Regulating excitability of peripheral afferents: emerging ion channel targets. Nat. Neurosci. 17, 153-163. doi: $10.1038 / \mathrm{nn} .3602$

Wu, J. X., Yuan, X. M., Wang, Q., Wei, W., and Xu, M. Y. (2016). Rho/ROCK acts downstream of lysophosphatidic acid receptor 1 in modulating P2X3 receptor-mediated bone cancer pain in rats. Mol. Pain 12, 1-10 doi: 10.1177/1744806916644929

Yung, Y. C., Stoddard, N. C., and Chun, J. (2014). LPA receptor signaling: pharmacology, physiology, and pathophysiology. J. Lipid Res. 55, 1192-1214. doi: 10.1194/jlr.R046458

Yung, Y. C., Stoddard, N. C., Mirendil, H., and Chun, J. (2015). Lysophosphatidic Acid signaling in the nervous system. Neuron 85, 669-682. doi: 10.1016/j.neuron.2015.01.009

Zamponi, G. W., Lewis, R. J., Todorovic, S. M., Arneric, S. P., and Snutch, T. P. (2009). Role of voltage-gated calcium channels in ascending pain pathways. Brain Res. Rev. 60, 84-89. doi: 10.1016/j.brainresrev.2008. 12.021

Conflict of Interest Statement: The authors declare that the research was conducted in the absence of any commercial or financial relationships that could be construed as a potential conflict of interest.

Copyright (c) 2018 Juárez-Contreras, Rosenbaum and Morales-Lázaro. This is an open-access article distributed under the terms of the Creative Commons Attribution License (CC BY). The use, distribution or reproduction in other forums is permitted, provided the original author(s) and the copyright owner(s) are credited and that the original publication in this journal is cited, in accordance with accepted academic practice. No use, distribution or reproduction is permitted which does not comply with these terms. 